\title{
Central de Ventiladores Mecânicos: Organização, Segurança e Qualidade*
}

\section{Central of Mechanical fan: Organization, Safety and Quality}

\author{
Miranildes de Abreu Batista', Erikson Custódio Alcântara², Lilian Khellen Gomes de Paula ${ }^{3}$
}

\section{RESUMO}

JUSTIFICATIVA E OBJETIVOS: A central de ventiladores mecânicos é a unidade do hospital com finalidade de organizar recursos de ventilação, promovendo controle e manutenção preventiva e organizacional destes equipamentos. $\mathrm{O}$ objetivo deste estudo foi elaborar uma proposta de implantação de uma central de ventiladores mecânicos em hospital universitário, subsidiado pela identificação do conhecimento técnico científico dos enfermeiros sobre o tema ventilação mecânica e pela detecção de problemas oriundos do gerenciamento descentralizado dos ventiladores.

MÉTODO: Trata-se de estudo descritivo exploratório com abordagem quantitativa, realizado com 13 enfermeiros de unidades de terapia intensiva. As informações foram coletadas através de entrevistas estruturadas e submetidas a análise descritiva do conteúdo.

RESULTADOS: Revelaram que os enfermeiros possuem dúvidas diversas, fato evidenciado por $100 \%$

1. Enfermeira Assistencial da Unidade de Terapia Intensiva do HCUFG. Professora, Mestre do Curso de Enfermagem da Universidade Paulista/GO.

2. Fisioterapeuta. Professor Mestre do Centro de Estudos Avançados em Fisioterapia da Universidade Católica de Goiás. Supervisor do Serviço de Fisioterapia Cardiorrespiratória e Fisioterapia em Unidade de Terapia Intensiva da Santa Casa de Misericórdia de Goiânia.

3. Fisioterapeuta. Graduada pela Universidade Católica de Goiás.

*Recebido das Unidades de Terapia Intensiva do Hospital das Clínicas da Universidade Federal de Goiás (HC-UFG), Goiânia, GO

- Estudo apresentado como exigência parcial para obtenção do Título de Especialista em Ventilação Mecânica pelo CEAF/UCG.

Apresentado em 27 de setembro de 2007.

Aceito para publicação em 06 de dezembro de 2007

Endereço para correspondência:

Lilian khellen G. de Paula

Rua 240, Quadra 7 - Lote 10 - Vila Montecelli

74655-180 Goiânia, GO

Fones: (62) 3203-36646 / (62) 8454-9758

E-mail: liliankhellen@hotmail.com

(C)Associação de Medicina Intensiva Brasileira, 2007 dos entrevistados que mencionaram a necessidade de cursos de capacitação voltados para a assistência de enfermagem ao paciente em ventilação mecânica. As situações descritas pelos enfermeiros no cotidiano, demonstraram que a descentralização do gerenciamento dos ventiladores mecânicos mostraram-se ineficaz quanto à organização, segurança e qualidade. A proposta de implantação de uma central de ventiladores aponta para melhorias na assistência, na formação de recursos humanos e na produção do conhecimento.

CONCLUSÕES: O perfil atual pode ser mudado através do rompimento de paradigmas institucionais e da instituição de práticas inovadoras que reforçarão o propósito de um hospital de grande porte voltado para o ensino, a pesquisa e a extensão.

Unitermos: assistência de enfermagem, central de ventiladores mecânicos, ventilação mecânica, UTI.

\section{SUMMARY}

BACKGROUND AND OBJECTIVES: The headquarters of mechanical fans is the unit of the hospital with purpose of organizing ventilation resources promoting control and preventive maintenance and organizational of these equipments. The objective of this study was to elaborate a proposal of implantation of a headquarters of mechanical fans in an academical hospital, subsidized by the identification of the male nurse's scientific technical knowledge on the theme ventilation mechanics and for the detection of problems originating from of the decentralized administration of the fans.

METHODS: It is treated of exploratory descriptive study with quantitative approach, accomplished with 13 male nurses of ICU. The information was collected through structured interviews and submitted the descriptive analysis of the content.

RESULTS: The results reveal that the male nurses possess several doubts, fact evidenced by $100 \%$ of the interviewees that mentioned the need of training courses gone back to the nursing attendance to the patient in 
ventilation mechanics. The situations described by the male nurses in the daily they demonstrate that the decentralization of the administration of the mechanical fans is shown ineffective as the organization, safety and quality. The proposal of implantation of a headquarters of fans appears for improvements in the attendance, in the formation of human resources and in the production of the knowledge.

CONCLUSÕES: It is ended that the current profile can be changed through the breaking of institutional paradigms and of the institution of innovative practices that you/they will reinforce the purpose of a hospital of great load gone back to teaching, he/she researches and extension.

Key Words: central of mechanical fans, ICU, nursing attendance, ventilation mechanics.

\section{INTRODUÇÃO}

Atualmente convive-se com os avanços tecnológicos e científicos que tem refletido no contexto evolutivo dos ventiladores mecânicos, desde os parâmetros pressométricos aos microprocessados ${ }^{1}$. De acordo com uma percepção futura, estratégica e inovadora surge a necessidade de interpretar aspectos que envolvem a prestação de serviço e suas nuances na qualidade da assistência em saúde.

A oferta de bons produtos e serviços, de maneira que garanta aos clientes deste serviço, segurança e qualidade, reduz os custos institucionais e é traduzido, na área da saúde, como busca pela qualidade total.

Há princípios que devem nortear um serviço que almeja práticas de excelência em suas ações: melhorar a atenção ao paciente, utilização criteriosa de seus recursos e treinamento de recursos humanos.

A estruturação de um serviço demonstra como funciona o sistema assistencial incluindo recursos materiais (instalações, equipamentos, recursos financeiros, remunerações), recursos humanos, (número e qualificação dos profissionais) e estrutura organizacional (organização da equipe, métodos e critérios operacionais, formas de avaliação e auditoria e remuneração dos serviços) $)^{2}$.

As metas estabelecidas, a curto e em longo prazo, devem ser mensuráveis e analisáveis, de maneira, a orientar planos de ação, estratégias de organização e implementação de melhorias. Com o intuito de otimizar os recursos e a necessidade de restaurar a saúde, a Unidade de Terapia Intensiva (UTI) especializa-se em procedimentos e tratamentos complexos que exigem pessoal capacitado interagindo com a complexidade tecnológica do momento, com reflexo no processo diagnóstico-terapêutico ${ }^{3}$.

A ventilação mecânica constitui um dos pilares terapêuticos que vem se mostrando como uma das principais ferramentas no tratamento de pacientes graves, em especial, os que apresentam insuficiência respiratória ${ }^{4,5}$. Este aspecto tem impulsionado os hospitais, principalmente os de grande porte, a se adequarem às potencialidades, limites e riscos da assistência ventilatória mecânica.

No emprego da ventilação mecânica, os profissionais e os procedimentos estão envolvidos na busca de soluções terapêuticas ${ }^{4}$. Cabe ao hospital, organizar o serviço de gerenciamento destes equipamentos, estabelecendo critérios que garantam o manuseio correto dos ventiladores através de procedimentos, preventivos e corretivos, envolvendo, neste contexto, todos os profissionais que, de uma maneira direta ou indireta, contribuem na busca pela qualidade da assistência ao paciente.

Na realidade a revisão conceitual no âmbito da assistência ventilatória conduz à consecução de soluções que melhorem o tratamento e evitem iatrogenias ${ }^{6}$.

O Hospital das Clínicas da Universidade Federal de Goiás (HC/UFG) é uma instituição de grande porte e alta complexidade, que atende múltiplas especialidades e presta atendimento pelo Sistema Único de Saúde (SUS). Apresenta três UTI sendo a clínica, a cirúrgica e a neonatal, onde os ventiladores mecânicos fazem parte do cenário.

O número de vagas de UTI no HC/UFG é desproporcional à demanda, gerando conflitos revelados através de pacientes que são submetidos à assistência ventilatória mecânica em unidades desprovidas de suporte para o paciente crítico. Portanto, o contexto que envolve a ventilação mecânica ultrapassa as paredes das UTI e se expande para outras unidades do hospital. Considera-se, também, a utilização de ventiladores para o transporte de pacientes.

O HC/UFG possui diversos ventiladores mecânicos, de diferentes fabricantes, do mais simples como o Bird Mark $7^{\circledR}$ ao mais rico em recursos como o Bennet $840^{\circledR}$. Esses ventiladores estão distribuídos em várias unidades do hospital, principalmente nas UTI; cada unidade é responsável pelo gerenciamento do seu equipamento. A relação número de leitos / ventiladores mecânicos é adequada ao número mínimo preconizado pela portaria que regulamenta o funcionamento das Unidades de Terapia Intensiva no Brasil. 
Percebe-se que a descentralização do conjunto destes equipamentos tem gerado quebra e perda de acessórios, manuseio inadequado do equipamento, culminando em freqüentes reparos dos ventiladores mecânicos no Setor de Engenharia Hospitalar do HC, gerando custos à instituição já que se trata de uma assistência técnica especializada.

A ausência de uma Central de Ventiladores Mecânicos dificulta a ação dos profissionais de saúde que se tornam impotentes diante de uma situação de intubação iminente sem ventilador disponível em sua unidade ou pela falta de peças no próprio circuito do ventilador mecânico, além de freqüente não verificação prévia destes equipamentos, expondo o usuário deste serviço a maiores riscos. Às vezes, por "ironia", pode ser que haja, em outra unidade, um ventilador disponível. No avanço da terapia intensiva, os ventiladores artificiais assumem papel vital no cuidado de pacientes críticos. A dependência dos pacientes que necessitam de assistência ventilatória torna necessária o bom funcionamento destes equipamentos ${ }^{1}$.

O paciente que necessita do uso de um ventilador mecânico necessita de uma assistência de enfermagem atenta e habilidosa ${ }^{7}$. Para que a atuação do enfermeiro seja eficiente, suas ações devem estar fundamentadas no método científico, através da aplicação do processo de enfermagem (sistematização da assistência de enfermagem). Ao elaborar um plano assistencial para pacientes em ventilação é fundamental que o enfermeiro conheça conceitos básicos de fisiologia pulmonar, bem como a importância de sua atuação profissional , com ênfase na prevenção de complicações ${ }^{8}$.

Diante do exposto, o estudo justificou-se pelas diversas problemáticas descritas e na necessidade de proporcionar segurança, organização e qualidade aos pacientes que necessitam de suporte ventilatório e teve como objetivo elaborar uma proposta de implantação de uma central de ventiladores mecânicos para o Hospital das Clínicas/UFG como meio prático, seguro e organizado de gerenciamento do uso racional dos ventiladores. O processo de Acreditação Hospitalar no $\mathrm{HC}$ prossegue e esta pesquisa visa contribuir com a implementação de melhorias que otimizem a qualidade assistencial.

Ressalta-se que a instituição de saúde que adota medidas inovadoras de acreditação revela responsabilidade e compromisso com a segurança e ética profissional, com os procedimentos que realiza e com a garantia da qualidade do atendimento ao paciente ${ }^{9}$.

\section{MÉTODO}

Após a aprovação pelo Comitê de Ética em Pesquisa do Hospital das Clínicas da Universidade Federal de Goiás, elaborou-se um estudo descritivo exploratório com abordagem quantitativa visando identificação de características do contexto da enfermagem na ventilação mecânica em UTI dessa Instituição.

O grupo participante do estudo contou com 13 enfermeiros que atuam em UTI (clínica, cirúrgica e neonatal) do Hospital das Clínicas da UFG. Foram excluídos do estudo, os enfermeiros de unidades que não possuem ventiladores mecânicos, os profissionais que estavam de férias ou licença médica, os que estavam afastados para capacitação, bem como aqueles que se recusaram a participar da entrevista.

As informações foram coletadas mediante entrevista conduzida por questionário contendo perguntas fechadas. Os dados foram analisados na forma descritiva. Os resultados apresentados através da busca de temas ou regularidades recorrentes nos dados onde aqueles conceitualmente significativos foram agrupados.

O projeto foi aprovado pelo Comitê de Ética em Pesquisa do Hospital das Clínicas da Universidade Federal de Goiás. Os enfermeiros foram esclarecidos sobre a finalidade do estudo e, quando de acordo, assinaram o Termo de Consentimento Livre e Esclarecido.

\section{RESULTADOS}

Observou-se que $92,3 \%$ dos profissionais participantes do estudo apresentaram dúvidas quanto aos riscos oriundos da assistência através de ventiladores mecânicos e $84,6 \%$ em relação ao ajuste de parâmetros básicos e $46,1 \%$ sobre as modalidades ventilatórias, mencionando a necessidade de mais esclarecimentos sobre o assunto.

No que se refere ao significado do ajuste de alarmes e a sua interpretação, quando acionado, $38,5 \%$ admitiram dúvidas sobre o tema. Dentre os entrevistados $38,5 \%$ referiram incertezas quanto ao uso e finalidades dos acessórios do ventilador mecânico. Hesitação quanto aos procedimentos de limpeza, de desinfecção e de esterilização de componentes do ventilador mecânico foi apontado por $23 \%$ dos participantes do estudo (Tabela 1). 
Tabela 1 - Dúvidas Apresentadas pelos Enfermeiros em Relação ao Contexto da Ventilação Mecânica $(n=13)$

\begin{tabular}{lcc}
\hline Questionamentos & $\mathrm{N}$ & $\%$ \\
\hline Modalidades ventilatórias & 6 & 46,1 \\
Finalidades dos acessórios do ventilador mecânico & 5 & 38,5 \\
Ajustes básicos para teste do ventilador mecânico & 11 & 84,6 \\
Montagem do circuito do ventilador mecânico & - & - \\
Significado dos alarmes & 5 & 38,5 \\
Riscos da ventilação mecânica & 12 & 92,3 \\
Processamento de materiais do ventilador mecânico & 3 & 23,0 \\
\hline
\end{tabular}

Indisponibilidade de um ventilador mecânico no momento "certo" foi referida por $92,3 \%$ dos enfermeiros, sendo que $46,1 \%$ dos entrevistados mencionaram terem assistido o paciente sem o ventilador mecânico e tê-lo ventilado com "ambú" até que se providenciasse um ventilador.

A inoperância do ventilador mecânico durante a sua instalação no paciente foi relatado por $92,3 \%$ dos enfermeiros. Ocasiões de trocas de ventiladores entre pacientes que necessitavam de recursos que outro ventilador dispunha foram referidos por $84,6 \%$ dos entrevistados. Insegurança sobre o papel da enfermagem no contexto da ventilação mecânica foi relatada por $46 \%$ dos participantes.

Dos participantes $53,8 \%$ referiram ocasião em que recebeu o ventilador, de outro plantão, montado de forma incorreta e 30,7\% referiram circunstâncias em que houve dificuldade de reconhecimento do sistema áudio visual acionado no ventilador mecânico (Tabela 2).

Tabela 2 - Situações Ocasionais Apontadas pelos Enfermeiros em seu Contexto Diário

\begin{tabular}{|c|c|c|}
\hline Situações & $\mathrm{N}$ & $\%$ \\
\hline Pediu o ventilador emprestado em outra unidade; & 12 & 92,3 \\
\hline Instalou o ventilador no paciente e ele não funcionou & 12 & 92,3 \\
\hline $\begin{array}{l}\text { Trocou ventiladores de pacientes, pois um dos pa- } \\
\text { cientes precisava ser ventilado com recursos que } \\
\text { outro equipamento possuía }\end{array}$ & 11 & 84,6 \\
\hline $\begin{array}{l}\text { Ventilou o paciente com "ambú" por um determina- } \\
\text { do tempo até que se providenciasse um ventilador } \\
\text { em outro setor do hospital }\end{array}$ & 06 & 46,1 \\
\hline $\begin{array}{l}\text { Recebeu ventilador mecânico montado de forma } \\
\text { incorreta }\end{array}$ & 7 & 53,8 \\
\hline $\begin{array}{l}\text { Não reconheceu o significado do alarme acionado } \\
\text { no ventilador mecânico }\end{array}$ & 4 & 30,7 \\
\hline $\begin{array}{l}\text { Sentiu insegurança em relação ao papel da enfer- } \\
\text { magem no contexto da ventilação mecânica }\end{array}$ & 6 & 46,1 \\
\hline $\begin{array}{l}\text { Sentiu falta de cursos de capacitação em ventilação } \\
\text { mecânica para enfermagem }\end{array}$ & 13 & 100 \\
\hline
\end{tabular}

\section{DISCUSSÃO}

A atuação do enfermeiro, no contexto da assistência ventilatória, é colocada como de intensa participação por tratar-se de um agente que assiste de forma contínua envolvendo desde o momento da instalação da via aérea artificial até a reabilitação do paciente ${ }^{8,10}$.

Neste estudo, solicitado aos enfermeiros para relacionar as principais dúvidas em relação à enfermagem no contexto da ventilação mecânica, os tópicos variaram desde o significado das modalidades ventilatórias até o processamento de material utilizado na assistência ventilatória.

Observou-se que os profissionais participantes quase em sua totalidade apresentaram dúvidas quanto aos riscos advindos da assistência através de ventiladores mecânicos, fato este seguido por dúvidas em relação ao ajuste de parâmetros básicos como modos de ventilação, $\mathrm{FiO}_{2}$, pressão inspiratória, pressão positiva ao final da expiração, volume corrente e freqüência respiratória.

Considerando tamanha importância, um ventilador mecânico antes de ser colocado em uso deve ter ajustes básicos pré-estabelecidos para se verificar o bom funcionamento do equipamento, papel este, desempenhado pelo enfermeiro, em seu contexto diário, em unidades de emergência. $O$ ventilador deve ser revisado quanto à montagem correta do circuito e quanto ao seu perfeito funcionamento, conectado a um pulmão teste, com comprovação de programação e leitura ${ }^{1,6}$. $\mathrm{O}$ estudo revelou dúvidas referentes às modalidades ventilatórias, mas acredita-se que seja uma realidade freqüente nas diversas UTI no Brasil. A assistência ventilatória envolve todos os profissionais que, de forma direta ou indireta, participam deste contexto e contribuem para a boa performance dos ventiladores artificiais em sua interação com o paciente, evitando as possíveis lesões causadas por ventilação mecânica.

A imprecisão no que se refere ao significado dos ajustes e a interpretação dos alarmes pode ser explicada pelo fato da literatura ser confusa nas descrições dos mesmos e a pouca relevância que se dá a este assunto tão importante. Os alarmes avisam sobre defeitos no sistema de ventilação ou alterações ventilatórias no paciente que, segundo análise do nível de prioridade, determinará a intervenção da equipe ${ }^{1}$.

A necessidade de modernização na prestação da assistência ventilatória propiciou a expansão e a diversificação de acessórios. Os ventiladores mecânicos são acompanhados de acessórios que garantem umidificação, aquecimento, inalação, coleta de líquidos, retenção de resíduos, sensoriamento e outros. Embora a maior parte da assistência de enfermagem esteja centrada no cuidado direto ao paciente, vale ressal- 
tar que também é de responsabilidade desta equipe o processamento dos materiais utilizados na assistência ventilatória já que pneumonia associada à ventilação mecânica é a infecção mais comum nas UTI, girando em torno de $86 \%$ dos casos de pneumonia hospita$\operatorname{lar}^{10,11}$.

Embora existam circuitos descartáveis, o seu alto custo exige o processamento deste material mediante normas institucionais, geralmente estabelecidas pela Comissão de Controle de Infecção Hospitalar $(\mathrm{CClH})^{4}$. Em relação à montagem do circuito do ventilador mecânico todos os enfermeiros afirmaram certeza no referido procedimento. Ressalta-se que conexões incorretas impedem o funcionamento adequado do aparelho, com conseqüências potencialmente desastrosas para o paciente ${ }^{12}$. Cabe ao enfermeiro montar o circuito ventilador-paciente com técnica asséptica ${ }^{8}$.

\section{Perfil Descentralizado}

Nas UTI a assistência tem se alicerçado no aparato tecnológico. A organização de equipamentos e/ ou procedimentos são eventos alteráveis conforme a cultura local. Os enfermeiros participantes do estudo revelaram situações ocasionais vivenciadas no contexto diário em que a descentralização dos ventiladores mecânicos mostra-se ineficaz quanto à organização, segurança e qualidade.

Peculiaridades do contexto descentralizado são retratadas em um quantitativo expressivo de situações que, apesar de tornarem-se internalizadas como "normais" no cotidiano das UTI, revelam-se como fatores geradores de estresse.

Os profissionais envolvidos no manuseio e na manutenção de ventiladores mecânicos devem receber treinamento específico e o enfermeiro como integrante da equipe de assistência ininterrupta deve ter conhecimento técnico-científico em relação ao significado dos alarmes, uma vez que qualquer função de entrada ou controle falhar poderá ocorrer uma situação potencialmente letal ${ }^{13}$.

Embora, na tabela 1, todos os enfermeiros tenham mencionado segurança quanto ao ato de montar o circuito do ventilador mecânico, na tabela 2 mais da metade admitiram receber o ventilador, de outro plantão, montado de forma incorreta na tabela 2. Surge a questão: qual membro da equipe de enfermagem vem executando o procedimento de montagem dos circuitos? Se a tarefa é delegada, a supervisão do enfermeiro é extremamente necessária para aferir a segurança imprescindível no processo de cuidar.
Em unanimidade, os enfermeiros reconheceram a deficiência oriunda da ausência de cursos de capacitação em assistência de enfermagem ao paciente em ventilação mecânica e a conseqüência óbvia é demonstrada na insegurança de $46 \%$ dos participantes no que diz respeito ao papel da enfermagem no contexto da ventilação mecânica.

As situações citadas envolvem os reflexos da descentralização dos ventiladores mecânicos: falta de procedimentos que envolvam controle de qualidade; descontrole sobre o número de ventiladores mecânicos existentes no hospital (em uso, prontos para uso, em manutenção dentro ou fora do hospital), diversidade de intermediadores entre equipe e assistência técnica; carência de acessórios devidamente requisitados; ausência de cursos de capacitação voltados, especificamente, para enfermagem; ausência de protocolos na assistência ventilatória; indefinição de papéis e competências.

Salienta-se que a vida de um paciente, em um hospital, depende da assistência multidisciplinar aliado à qualidade e disponibilidade de recursos materiais, humanos e financeiros ${ }^{14}$.

\section{Central de Ventiladores Mecânicos - Reflexos da Inovação}

A Central de Ventiladores Mecânicos seria a unidade do Hospital das Clínicas/UFG com finalidade de organizar recursos de ventilação promovendo controle e manutenção preventiva e organizacional destes equipamentos. Atenderia as UTI, Pronto Socorro e demais unidades que, porventura, venham a assistir pacientes em instabilidade ventilatória. Promoveria integração entre profissionais da saúde que assistem o paciente de maneira direta como médicos, enfermeiros, fisioterapeutas, nutricionistas e, de forma indireta, engenheiros e técnicos em engenharia hospitalar.

Esse estudo trata-se de um projeto de implantação de um novo serviço que busca o aprimoramento de práticas e rotinas que tragam benefícios que elevem o padrão de qualidade assistencial.

Por ser de um hospital universitário, além das atividades assistenciais, a equipe da Central de Ventiladores teria um importante papel na formação de recursos humanos e na produção de conhecimento na área da ventilação mecânica, quesitos que refletem reconhecimento de excelência na assistência.

\section{Fluxograma}

Atividades da Central de Ventiladores: educativas (or- 
ganização e coordenação de cursos de ventilação mecânica), assistenciais (incluem o preparo do ventilador mecânico, o acompanhamento do paciente em ventilação, a troca do circuito e processamento do material) e gerenciais (controle sobre o funcionamento da unidade; controle de registro de localização, tempo de uso por paciente, equipamento em manutenção dentro ou fora das instalações do hospital; controle de troca de circuitos e gerenciamento de custos).

Por tratar-se de um hospital de ensino a central de ventiladores torna-se campo de aprendizagem para disciplinas de diversas graduações (enfermagem, medicina, fisioterapia) e residências que envolvam cuidados com o paciente crítico e de alta complexidade.

\section{Fluxograma}

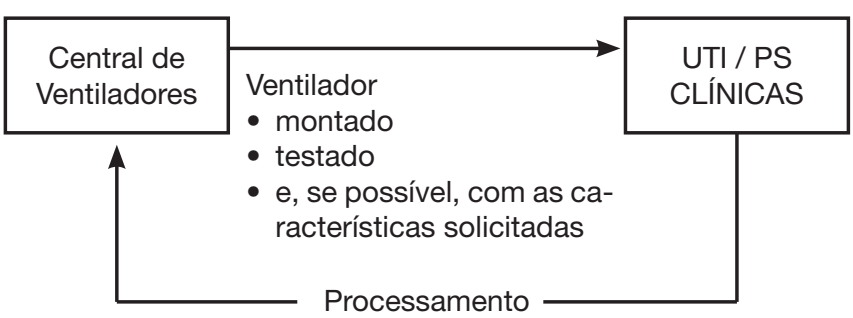

\section{CONCLUSÃO}

Ficaram caracterizadas as necessidades de enfermeiros no campo da ventilação mecânica em meio à repressão do cotidiano tumultuado de um hospital de grande porte.

Os rompimentos de paradigmas institucionais através de atitudes inovadoras reforçam os propósitos de um hospital universitário voltado para ensino, pesquisa e extensão.

Dentre as questões levantadas, no grupo de enfermeiros deste estudo, podemos inferir que grande parte apresenta dúvidas em ajustes iniciais do ventilador mecânico e que a dificuldade em relação à ventilação mecânica é comum a todos os enfermeiros entrevistados.

Este estudo permitiu-nos observar pontos conflitantes no dia-a-dia da UTI no que diz respeito à viabilização da assistência ventilatória que, atualmente, gira em torno das desvantagens da descentralização dos ventiladores mecânicos.

A proximidade com esta realidade levam a questionar o modelo atual de gerenciamento descentralizado dos ventiladores e sugerir mudanças neste contexto com a implantação de um novo serviço: a Central de Ventiladores Mecânicos - com a filosofia de oferecer assistência ventilatória com qualidade ao paciente, reflexo este, do gerenciamento eficaz do uso dos ventiladores, da atualização multiprofissional e da educação continuada em serviço.

\section{REFERÊNCIAS}

01. David CM - Ventilação mecânica - Da Fisiologia à Prática Clínica. Rio de Janeiro, Revinter, 2001.

02. Donabedian A - Quality assessment an assurance: unity of purpose, diversity of means. Inquiry 1988;25:173-192.

03. Dodson B Jr - Facilities in Clinical Engineering: Principles and Practices. Englewood Cliffs: Practice-Hall, 1979.

04. Zuñiga QGP - Ventilação Mecânica Básica para Enfermagem. São Paulo: Atheneu, 2004.

05. Damasceno MPCD, David CMN, Souza PCSP et al - Ventilação mecânica no Brasil. Aspectos epidemiológicos. RBTI, 2006;18:219-228.

06. Benito S, Net A - Ventilação Mecânica. Rio de Janeiro: Revinter, 2002.

07. Hudak CM, Gallo BM - Cuidados Intensivos de Enfermagem. Uma Abordagem Holística. 6a Ed, Rio de Janeiro: Guanabara-Koogan, 1997.

08. Auler JOC, Amaral RVG - Assistência Ventilatória Mecânica. São Paulo: Atheneu, 1995.

09. ONA. Organização Nacional de Acreditação. Manual das Organizações Prestadoras de Serviços Hospitalares. Porto Alegre: Educat, 2004.

10. Barbosa SFF, Dopico L, Canuto A et al - Papel da enfermagem na assistência ao paciente em ventilação mecânica. J Bras de Pneumol, 2000;26:24-27.

11. Guimarães MM, Rocco JR - Prevalence of ventilador-associated pneumonia in a university hospital and prognosis for the patients affected. $J$ Bras Pneumol, 2006;32:339-346.

12. Maia JA - Ventilação Pulmonar Artificial. São Paulo: Atheneu, 2002.

13. Graig LS, Wilkins RL, Stoller JK - Fundamentos da Terapia Respiratória $7^{\mathrm{a}}$ Ed, São Paulo: Manole, 2000.

14. Madureira CR, Veiga K, Sant'ana AF - Management of technology in intensive care. Rev Lat Am Enfermagem. 2000;8:68-75. 